\title{
Empirical Mode Decomposition of Throat Microphone Recordings for Intake Classification
}

\author{
M. A. Tuğtekin Turan \\ Multimedia, Vision and Graphics Laboratory \\ Koç University \\ College of Engineering \\ Istanbul, Turkey \\ mturan@ku.edu.tr
}

\author{
Engin Erzin \\ Multimedia, Vision and Graphics Laboratory \\ Koç University \\ College of Engineering \\ Istanbul, Turkey \\ eerzin@ku.edu.tr
}

\begin{abstract}
Wearable sensor systems can deliver promising solutions to automatic monitoring of ingestive behavior. This study presents an on-body sensor system and related signal processing techniques to classify different types of food intake sounds. A piezoelectric throat microphone is used to capture food consumption sounds from the neck. The recorded signals are firstly segmented and decomposed using the empirical mode decomposition (EMD) analysis. EMD has been a widely implemented tool to analyze non-stationary and non-linear signals by decomposing data into a series of sub-band oscillations known as intrinsic mode functions (IMFs). For each decomposed IMF signal, time and frequency domain features are then computed to provide a multi-resolution representation of the signal. The minimum redundancy maximum relevance (mRMR) principle is utilized to investigate the most representative features for the food intake classification task, which is carried out using the support vector machines. Experimental evaluations over selected groups of features and EMD achieve significant performance improvements compared to the baseline classification system without EMD.
\end{abstract}

\section{CCS CONCEPTS}

- Hardware $\rightarrow$ Sensor applications and deployments; • Social and professional topics $\rightarrow$ Medical technologies; $\bullet$ Applied computing $\rightarrow$ Health care information systems;

\section{KEYWORDS}

dietary monitoring; empirical mode decomposition; wearable sensor minimum redundancy maximum relevance; throat microphone; food intake classification

\section{INTRODUCTION}

Eating behavior and food consumption patterns contribute towards to the development of dietary monitoring, mental eating disorders

Permission to make digital or hard copies of all or part of this work for personal or classroom use is granted without fee provided that copies are not made or distributed for profit or commercial advantage and that copies bear this notice and the full citation on the first page. Copyrights for components of this work owned by others than the author(s) must be honored. Abstracting with credit is permitted. To copy otherwise, or republish, to post on servers or to redistribute to lists, requires prior specific permission and/or a fee. Request permissions from permissions@acm.org.

MMHealth'17, October 23-27, 2017, Mountain View, CA, USA

(C) 2017 Copyright held by the owner/author(s). Publication rights licensed to Association for Computing Machinery.

ACM ISBN 978-1-4503-5504-9/17/10 . \$ \$15.00

https://doi.org/10.1145/3132635.3132640 such as bulimia or anorexia nervosa [9] and other neurological impairments like dysphagia [3]. People suffering from obesity are also at a higher risk of blood pressure and other cardiovascular diseases [20]. According to World Health Organization (WHO), overweight and obesity are one of the main reasons for deaths spreading all over the world [16]. Automatic dietary monitoring can deliver an important tool for individuals to change their eating behavior.

Existing solutions of dietary monitoring such as food recall, foodfrequency questionnaires [12] and self-report diaries [13] achieve low accuracy as people have tendency to report biased consumption, which causes an inaccurate estimate of daily intake. Objective and automated methods of intake monitoring were introduced as a potential solution to replace the manual reporting procedures. These methods measure the periods of food intake with minimum participation so they provide deeper insight of eating behavior by improving the detection and classification accuracy.

Videofluoroscopy and electromyography (EMG) are considered as the benchmark methods in food intake studies. Videofluoroscopy requires detailed preparation with risks of aspiration and it must be performed in a clinic, while EMG is too invasive due to frequently used electrodes placed over the muscles of neck. Thus, new techniques have been used in recent years to develop alternative solutions for dietary assessment.

Chewing and swallowing are regarded as the two main stages of food intake mechanism. After the food is taken to the mouth, chewing performs consecutive food degradation to shape a food bolus that can be swallowed. Several systems have identified chewing and swallowing acoustically. For example in [17], acoustic data is acquired from multiple microphones coupled with strain gauge sensor from the ear canal. Wearing such a system is very unpleasant for the users in terms of daily usage. In contrast, we use a single necklace-like device to collect acoustic signals non-invasively, which is more convenient and comfortable. In another work, eatingrelated arm gestures were detected by accelerometers and gyroscopes [2]. Spoon, mug or hand usage associated with food intake were identified. However, subject-depended eating style may limit the usefulness of this approach.

Piezoelectric sensors, which produce an output voltage corresponding with the mechanical stress, are used in many on-body sensing applications. Recently, they have been applied to problems in the medical domain, such as identifying individual heart rates and respiration [11]. Very few works describe attempts to use piezoelectric sensors for monitoring food ingestion, with several 
exceptions [6], although the detection of swallowing disorders such as dysphagia was the primary objective of their work.

In this work, we use an oscillatory data investigation tool called as the Empirical Mode Decomposition (EMD) introduced in [10]. It has been a novel technique for analysis of non-linear and nonstationary time-series and recently used in various signal processing problems $[1,8,14]$. The EMD decomposes any arbitrary time-series signal into a set of components known as intrinsic mode functions (IMFs). IMFs hold different frequency scales due to the dyadic filter bank structure of the EMD [7].

Traditional speech processing systems have been using the sourcefilter theory of linear prediction for many years as a principal representation. This theory simulates the speech signal as an output of the vocal tract filter excited by a dynamic source. Although the speech and ingestion signals have different characteristics and production mechanism, both of them have non-stationary nature. Methods using short time Fourier transform (STFT) on the basis of linear prediction theory can extract the spectral information over short time windows (10-30 ms) alongside locally calculated Fourier transform.

We consider the EMD for the analysis of food ingestion signals rather than the conventional Fourier-based or source-filter approaches. One disadvantage of applying STFT to food ingestion recordings is the "a-priori" assumption of a linear and stationary model (i.e. sinusoidal amplitudes and constant frequencies are covering the whole signal). Therefore, STFT only provides fixed time and frequency resolution over short segments. In contrast to the STFT, EMD is an adaptive, data-driven and "a-posteriori" tool which does not need "a-priori" basis.

To solve the issues related to fixed division of STFT, wavelet transform (WT) have been developed around late 80's [4]. It can be considered as an alternative representation of Fourier-based procedure with adjustable window from which to replace sinusoidal function to mother wavelet. However, due to the additional complexity and dependency of mother wavelet selection, this transformation have not become so popular for non-stationary signal analysis. Considering food intake recordings, one of the main factors is the shortness and non-stationarity of total data segments. Therefore, Fourier-based spectral examination methods such as STFT and WT encounter limitations in comparison with EMD.

A significant problem of any classification scheme is to tackle with the increasing number of variables which may grow up the computational burden and mitigate the classification accuracy as well. The ultimate aim is to extract the optimum features from the signals that can perform best discrimination between groups. Feature reduction is the technique of selecting a subset of relevant features for building robust classification models. By removing most irrelevant and redundant features from the data, the classification problem becomes more feasible.

This study we utilized the minimum redundancy maximum relevance (mRMR) algorithm, which was originally proposed by Peng et al. to solve the feature selection problem of micro-array gene expression data [5]. It tends to select a subset of features having the maximum correlation within a class (relevance) and the minimum correlation between themselves (redundancy). Relevance can be calculated by using the F-statistic (for continuous features) or mutual information (for discrete features) and redundancy can be calculated using Pearson correlation coefficient or mutual information in a similar manner.

The goal of this study is to develop non-invasive monitoring and automated methods for classification of food intake. A methodology based on EMD tool is proposed to classify food intake from epochdivided sound signals. This work also explores the usefulness of EMD analysis for multi-class food intake classification along with the support vector machine (SVM) classifier. Monitoring of the food intake sound is conducted by a throat microphone sensor located over laryngopharynx. The mRMR feature selection process is utilized to determine the most relevant set of features on different IMFs representing the original sound.

The primary novelty of our work is the analysis of food intake sounds through the EMD and the investigation of most relevant features for food intake classification by means of the mRMR. Although a number of studies have been presented for the automatic food classification, to our knowledge no work has been done on EMD analysis for ingestion sounds. We propose a solution using a novel decomposition to accurately distinguish different food intake types.

The rest of this paper is organized as follows. Methods including EMD analysis, mRMR feature selection and SVM procedure are described in Section 2. We present our experimental setup with the evaluations in Section 3. The conclusion and general discussion are given in Section 4.

\section{METHODS}

Block diagram of the proposed food intake classification system is given in Figure 1. Throat microphone sound recordings are firstly processed through temporal windows of length $L_{W}$ with $50 \%$ overlaps. Feature extraction defines a set of features over all the IMFs and residual signal, where a reduced dimensional feature is then selected by the mRMR. Finally, we perform food intake classification for each data segment.

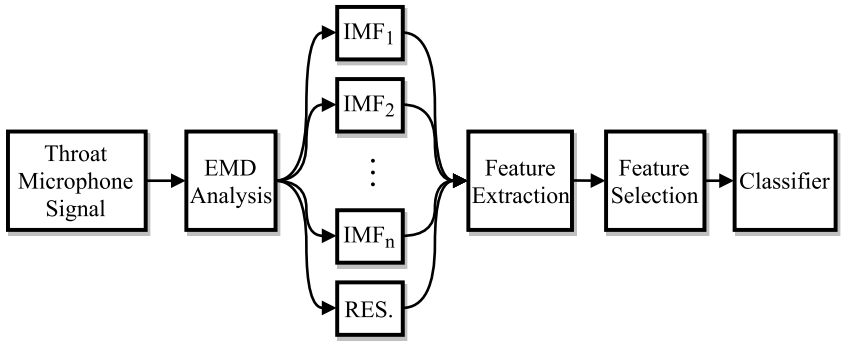

Figure 1: Block diagram of the proposed food intake classification system

\subsection{EMD analysis}

Throat microphones are skin-attached piezoelectric sensors that can record sound signals in the form of tissue vibrations. They can only capture low frequency bands, since tissue and bones act as a low-pass filter $[18,19]$. In this study, we investigate EMD-based decomposition of the ingestion sounds, which are captured through 

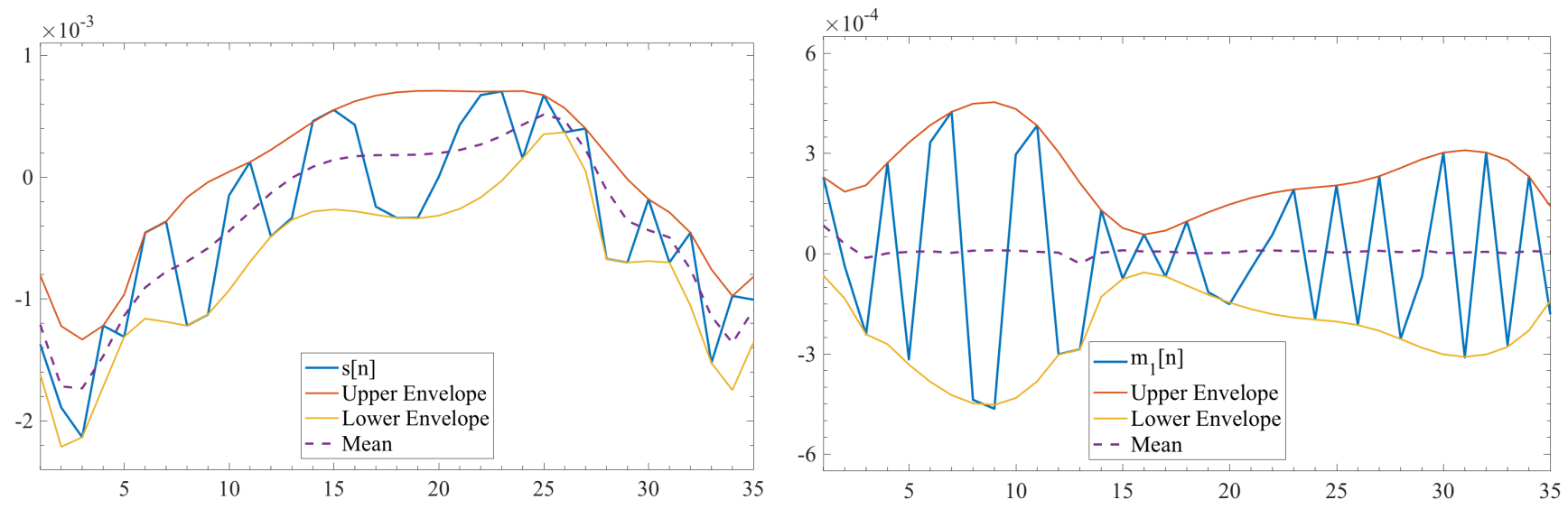

Figure 2: First iteration of sifting process (left), first IMF after repetition of the algorithm (right)

the throat microphones. EMD is a mathematical tool proposed by Huang [10]. It enables decomposition of non-linear and nonstationary data into dynamic basis functions known as IMFs. The decomposition is basically based on the simple assumption that any data consists of different sub-band modes of hierarchic oscillations. In principle, an IMF should satisfy the following two conditions:

(1) The number of extrema points and zero crossings are either equal or differ at most by one.

(2) The mean value of the envelopes defined by the local maxima and minima must be equal to zero.

Ultimately, the decomposition of a signal $s[n]$ as $K$ number of IMFs and a residual $r_{K}$ is given by

$$
s[n]=\sum_{k=1}^{K} m_{k}[n]+r_{K}[n] .
$$

The extraction of signal into different number of IMFs, $m_{k}[n]$, is known as sifting algorithm and summarized as the following:

(1) Identify all local maxima and minima for a given discrete time signal $s[n]$.

(2) Calculate the upper and lower envelopes, $E_{U}$ and $E_{L}$, using cubic spline interpolation that connects the local extrema.

(3) Find the mean value of the upper and lower envelopes, $E_{\mu}=$ $\left(E_{U}+E_{L}\right) / 2$ and subtract the mean from the original signal, $s[n] \triangleq s[n]-E_{\mu}$.

(4) Execute the last three steps considering the updated $s[n]$ as the input signal until it satisfies the IMF conditions described above.

(5) Subtract the first IMF, $m_{1}$, from $s[n]$ to obtain the residue $r_{1}[n]$. This signal becomes $s[n]$ for the next iteration.

(6) Iterate steps (1) to (5) on the residual $r_{k} ; k=2, \ldots, K$ to find all the IMFs of the initial signal.

The sifting algorithm has two aims: 1) to eliminate riding waves, and 2) to make the wave signals more symmetric. Ideally after the first round of algorithm, the resulting signal at step (3) should satisfy the definition of an IMF. However, a small perturbed curve can be broaden to become local extrema which generates new coordinates although the fitting is accurate. Therefore, whole process is repeated as many times as is required to achieve an IMF at the final step of sifting algorithm. A detailed segment of the first iteration and obtained corresponding IMF is depicted in Figure 2.

This procedure can be terminated intuitively either when the residual becomes smaller than the predetermined threshold value or when the residual becomes a monotonic function where no more IMF can be extracted. Figure 3 illustrates the generation of the first four IMFs and the residual obtained from decomposing a sample almond segment of $500 \mathrm{~ms}$ length.

Clearly, the highest oscillation is embedded in the first IMF. This is an expected output of the sifting process which is based on diminishing scheme of the largest period component from data. Consequently, the components with the highest frequencies are stored in the first IMF. In other words, lower frequency content will be more dominant with the increasing order of the IMFs.

Accurate estimation of the IMFs may become an important factor because of the interpolation errors or an erroneous stopping criteria. Since the sifting process is vulnerable to the small changes and the choice of stopping criteria may produce different IMFs, the overall EMD procedure is not unique.

Finally, each IMF represents a simple oscillation as a counterpart to the harmonic function with variable amplitude and frequency as functions of time instead of constant amplitude and frequency. Furthermore, the oscillations should also be symmetric as compared to the local mean. The signal may have many different modes of oscillations at any time instance where each one is superposing on the others. In all implementations, we use the EMD toolbox available on Flandrin's web page ${ }^{1}$.

\subsection{Feature extraction}

In this study, we pick 13 time domain and 3 frequency domain descriptors empirically to construct the feature vector for intake classification task. Note that extraction of these descriptors require minimal computational resources and they provide enough discrimination across different food intake events.

\footnotetext{
${ }^{1}$ http://perso.ens-lyon.fr/patrick.flandrin/emd.html
} 


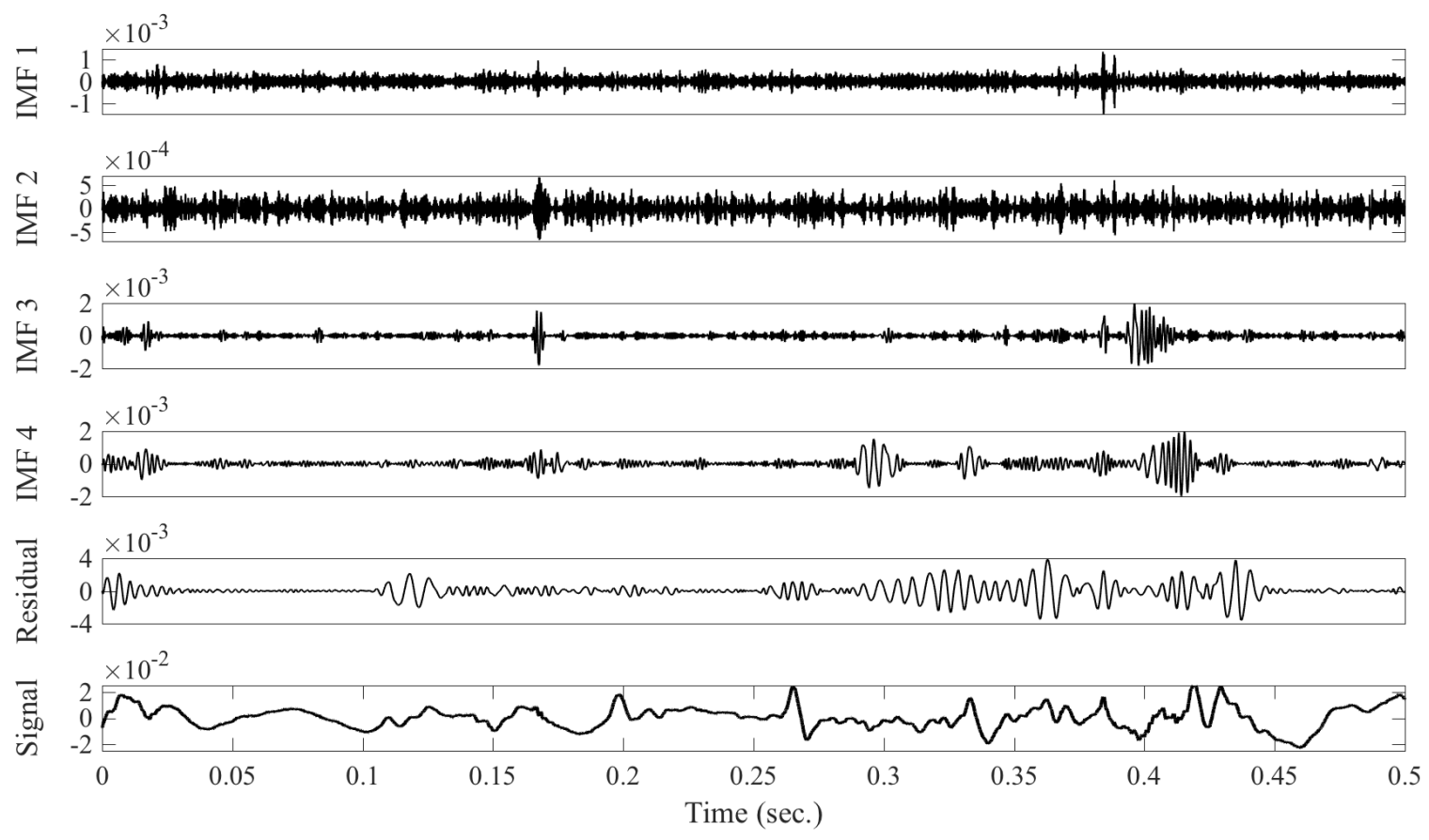

Figure 3: A sample empirical mode decomposition of a food intake signal

A summary of the feature set is given in Table 1 . The first 11 parameters are low-level statistical descriptors of the signal. Zerocrossing rate and short-time energy are one of the frequently used descriptors in time-series analysis that reveal time-varying structure easily. The last three frequency domain features are extracted from the power spectral density estimate based on Welch's averaging.

\subsection{Feature selection}

The features, as defined in the previous subsection, are repeatedly extracted over all the IMFs and the residual signal to define an extended feature set $\mathcal{X}$. In feature selection, we rank the descriptors in the extended feature set using the mRMR selection algorithm, which targets to rank features with a high correlation within the class and a low correlation between themselves. The mRMR is a

Table 1: Time and frequency domain descriptors in the feature set

\begin{tabular}{llcl}
\hline$\#$ & Description & $\#$ & Description \\
\hline 1 & Minimum Value & 9 & Third Quartile \\
2 & Maximum Value & 10 & Interquartile Range \\
3 & Mean Value & 11 & Range of Data \\
4 & Standard Dev. & 12 & Windowed Energy \\
5 & Kurtosis & 13 & Zero-Crossing Rate \\
6 & Skewness & 14 & Maximum Power \\
7 & First Quartile & 15 & Average Power \\
8 & Median Value & 16 & Freq. at Max. Power \\
\hline
\end{tabular}

greedy algorithm that ranks one feature at an iteration incrementally and considers only pairwise redundancies between features. Therefore, features that are maximally dissimilar are selected to form a smaller feature vector with minimum redundancy.

A way of measuring the minimum redundancy among feature space $\mathcal{X}$ with $n$ features $x_{i}, i=1, \ldots, n$ is,

$$
\frac{1}{|\mathcal{X}|^{2}} \sum_{x_{i}, x_{j} \in \mathcal{X}} I\left(x_{i}, x_{j}\right)
$$

where $I\left(x_{i}, x_{j}\right)$ denotes the mutual information of features $x_{i}, x_{j}$ and $|X|$ is the size of feature space.

The mRMR criteria targets to minimize redundancy over accumulated mutual information $\sum_{i \neq j} I\left(x_{i}, x_{j}\right)$ while maximizing relevance of $x_{i}$ within the target class $c$ over the mutual information $I\left(x_{i}, c\right)$. Since an exhaustive search for the best subset is impractical, the selected set should be obtained in an incremental way. Suppose we already have $\mathcal{X}_{n-1}$, the feature set with $n-1$ selected features. The task is to select $n$-th feature from the set $\mathcal{X}-\mathcal{X}_{n-1}$. This is done by selecting the $n$-th feature with the following maximization,

$$
\underset{x_{j} \in \mathcal{X}_{n-1}}{\arg \max }\left[I\left(x_{j}, c\right)-\frac{1}{n-1} \sum_{x_{i} \in \mathcal{X}_{n-1}} I\left(x_{i}, x_{j}\right)\right] .
$$

In this paper, we adapt the implementation of mRMR defined in [15], which effectively solves the global optimization problem of (3).

\subsection{Classifier}

We utilized the SVM classifier for the food intake classification. SVM classifiers attain robustness and high generalization prop- 
erties, which are useful when training group models with high variability between samples. It basically tries to find a hyperplane that maximizes the separation of margins between data points given a weight vector $\omega$ and bias $b$. In this study, SVM classifiers are trained with a radial-basis function (RBF) kernel that forms $K\left(x_{i}, x_{j}\right)=\exp \left(-\gamma\left\|x_{i}-x_{j}\right\|^{2}\right)$. The width parameter $\gamma$ is defined as $\gamma=1 / 2 \sigma^{2}$.

Our major interest is to reveal the discriminating capabilities of the proposed features through the EMD framework. The target here is much on the decomposition instead of the classifier type. Thus, one of the well-known classical techniques like SVM have been utilized.

\section{EXPERIMENTAL RESULTS}

\subsection{Food intake dataset}

We collect an in-house food intake sound dataset from a single subject using the IASUS NT3 throat microphone. The subject is a male and does not present any medical condition that would interfere with normal food intake. The IASUS NT3 throat microphone is placed over the trachea and sound recordings are captured at $16 \mathrm{kHz}$ sampling rate. Time waveform and spectrogram of a sample recording with $7 \mathrm{sec}$ duration are respectively given in the top and bottom plots in Figure 4.

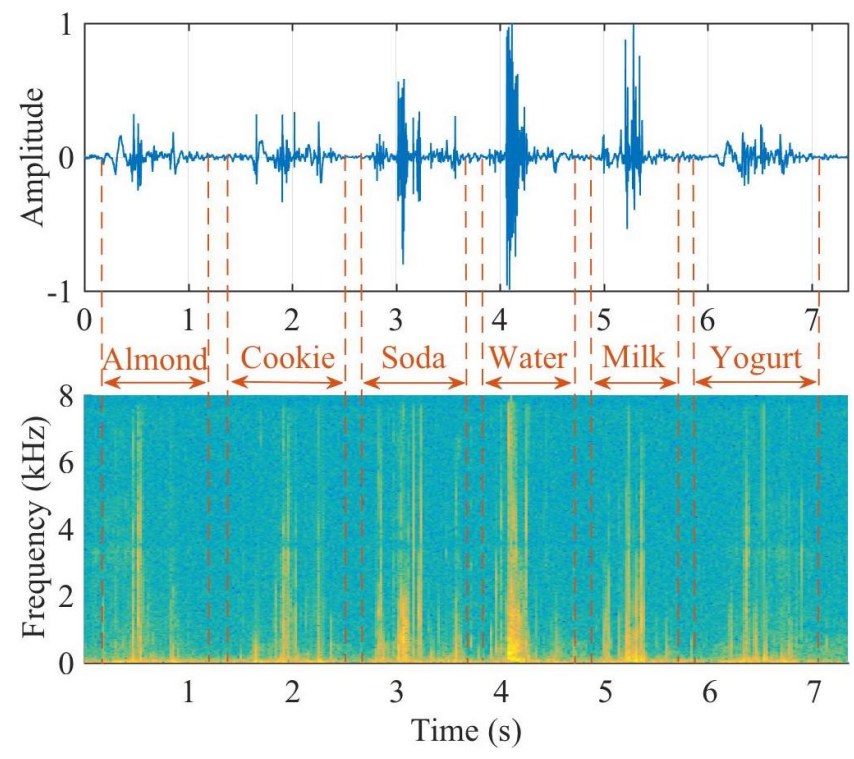

Figure 4: Waveform and spectrogram representations of different food intakes

The food items consumed during the intakes are selected to represent different physical properties of everyday foods such as "hardness" and "crunchiness". For example, cookie is a typical solid food and yogurt is a semi-solid food while almond is a hard crunchy food. The collected dataset includes a total of 481 recorded food intakes, which are summarized in Table 2.
Table 2: A summary of the food intake dataset

\begin{tabular}{cccc}
\hline Food Item & $\begin{array}{c}\text { Number of } \\
\text { Intakes }\end{array}$ & $\begin{array}{c}\text { Average } \\
\text { Duration }\end{array}$ & Viscosity \\
\hline Almond & 84 & $1.7 \pm 0.4 s$ & Solid \\
Cookie & 65 & $1.9 \pm 0.3 s$ & Solid \\
Soda Drink & 71 & $1.8 \pm 0.5 s$ & Liquid \\
Water & 95 & $1.5 \pm 0.3 s$ & Liquid \\
Milk & 87 & $1.7 \pm 0.4 s$ & Liquid \\
Yogurt & 79 & $1.6 \pm 0.5 \mathrm{~s}$ & Semi-Solid \\
\hline
\end{tabular}

\section{2 mRMR ranking evaluations}

The mRMR ranking of the features is applied both before and after the EMD processing for different experimental analysis. We limit EMD processing to extract up to four IMFs and a residual to reduce the computational cost. As suggested in [5], the mRMR-based feature selection performs better for quantized data as noise in continuous features could be minimized with discrete variables. We also choose to quantize continuous valued features into 16 discrete centroids prior to $\mathrm{mRMR}$ ranking. In order to define mRMR-ranked feature representations, let us define all available feature space as

$$
\mathcal{X}=\left\{x_{i, j}\right\} \text { for } i=1, \ldots, 16, j=0, \ldots, 5
$$

where $\left\{x_{i, 0}\right\}$ are the features extracted from the food intake sounds $s[n],\left\{x_{i, 5}\right\}$ are the features extracted from the residual in the EMD, and the features $\left\{x_{i, j}\right\}$ are from the first four IMF signals $m_{j}[n]$ for $j=1, \ldots, 4$.

We define four selected feature representations over the available feature space. The first feature set is the original food intake sounds, $\mathbf{F}_{S}=\left[x_{1,0} \cdots x_{16,0}\right]$. We then performed mRMR on this feature set and select the $n$ most relevant features and denote them as $\mathrm{F}_{S}^{n}$. The last two feature sets are defined over the decomposed signals through EMD. First, we rank the descriptors in the feature set, where each descriptor is a 5 dimensional vector (4 IMFs plus a residual) in the decomposed representation, $\mathbf{x}_{i}^{D}=\left[x_{i, 1} \cdots x_{i, 5}\right]$. The third feature set is defined over these mRMR ranked descriptor vectors by selecting the $n$ most relevant vectors as, $\mathrm{F}_{D}^{5 n}=\left[\mathbf{x}_{1^{*}}^{D} \cdots \mathbf{x}_{n^{*}}^{D}\right]$, where $\mathbf{x}_{i^{*}}^{D}$ is the $i$-th ranked descriptor vector. Finally, we perform mRMR on all components of the decomposed representation, $\left\{x_{1: 5,1: 16}\right\}$, and extract a ranked feature sequence, $\left\{x_{1^{*}} \cdots x_{80^{*}}\right\}$. The fourth feature set is defined as the $n$ most relevant feature components over the EMD derived features as, $\mathbf{F}_{M D}^{n}=\left[x_{1^{*}} \cdots x_{n^{*}}\right]$.

Figure 5 presents ranking plots of the all feature descriptors over the EMD components. Feature descriptors are also ordered from top to bottom in their mRMR rankings as used in $\mathrm{F}_{D}^{n}$. Note that range of data, mean, frequency at maximum power and zero crossing rate are the top four descriptors in the mRMR ranking. On the other hand, low order IMFs are observed more relevant than the higher IMFs and the residual.

\subsection{Classification evaluations}

We utilized four-fold cross validation to evaluate food intake classification performances. A temporal window length is chosen based on the following two criteria: 1) Portion length should preserves the 


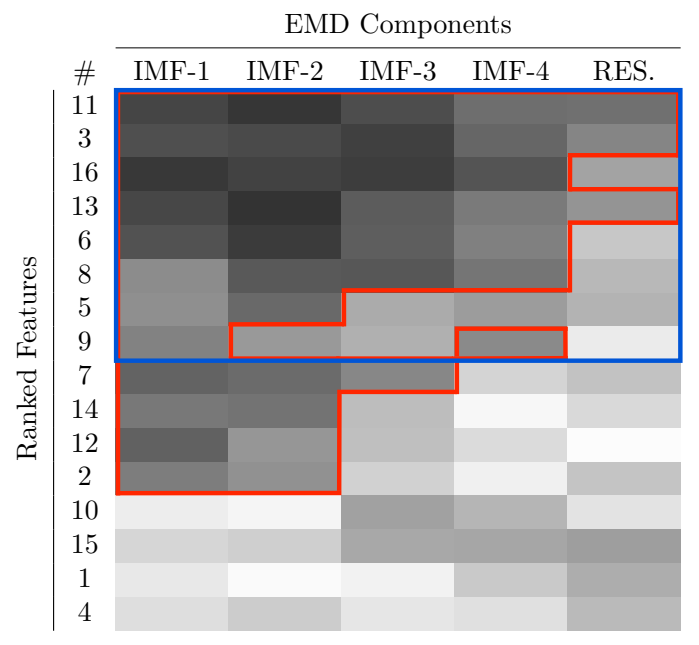

Figure 5: The mRMR ranking of the feature descriptors over the EMD components. Darker colors represent higher rankings.

non-stationarity behavior of the sensor signals, 2) There should be a fair comparison between classification accuracy and computational time required by the methodology. Based on this discussion, the intake data is processed and features are calculated over $500 \mathrm{~ms}$ overlapping segments with $50 \%$ overlap.

The classification accuracy is defined as the average of precision and recall rates. The precision and recall are defined as,

$$
\text { Precision }=\frac{T_{+}}{T_{+}+F_{+}} \quad, \quad \text { Recall }=\frac{T_{+}}{T_{+}+F_{-}},
$$

where the true positive $\left(T_{+}\right)$is the number of correctly classified intake windows, whereas the false positive $\left(F_{+}\right)$is the number of incorrectly classified windows and the false negative $\left(F_{-}\right)$is the number of times that classifier is failed to catch the successful intake events.

Table 3 shows a summary of the food intake classification accuracy results for the four selected feature sets as a function of the $n$ most relevant features after mRMR. The highest performance of each experiment is marked as bold for ease of visualization. The first feature set $\mathbf{F}_{S}$ is a baseline and sets a benchmark for the food intake classification. The mRMR-based selection over the baseline, $\mathrm{F}_{S}^{n}$,

Table 3: Classification accuracies of the selected feature sets.

Accuracy as feature dimension $n(\%)$

\begin{tabular}{lcccc}
\hline$n$ & 4 & 8 & 12 & 16 \\
\hline $\mathbf{F}_{S}$ & & & & $\mathbf{7 1 . 6}$ \\
$\mathbf{F}_{S}^{n}$ & 66.3 & 69.8 & $\mathbf{7 2 . 5}$ & 71.6 \\
\hline$n$ & 20 & 40 & 60 & 80 \\
\hline $\mathbf{F}_{D}^{n}$ & 76.7 & $\mathbf{7 7 . 3}$ & 74.9 & 72.8 \\
$\mathbf{F}_{M D}^{n}$ & 77.1 & $\mathbf{7 9 . 4}$ & 75.2 & 72.8 \\
\hline
\end{tabular}

attains some classification improvement with the 12 most relevant descriptors and achieves $72.5 \%$ accuracy.

Performance of the mRMR ranked descriptor vectors $\mathrm{F}_{D}^{n}$ is on the third row of the Table 3 . The $\mathrm{F}_{D}^{n}$ feature set achieves $77.3 \%$ accuracy with the 8 most relevant descriptor vectors for each component, which constructs a 40 dimensional representation (blue rectangular in Figure 5). The last row presents performance of the mRMR ranked features $\mathrm{F}_{M D}^{n}$, where the best classification accuracy is attained as $79.4 \%$ using the 40 most relevant features (red contour in Figure 5).

\section{DISCUSSION AND CONCLUSION}

In this paper, we have presented a food intake classification system from throat microphone recordings through the mRMR feature selection scheme over the EMD based signal decompositions. The employed sensor technology is non-invasive and not sensitive to acoustic noise.

EMD is a data-driven technique for non-linear and non-stationary signal analysis. The initial idea is to produce sub-band signals that represent local oscillations of the signal and to iterate the procedure on slower components hierarchically. After this decomposition method, a finite number of oscillatory components called as IMFs are extracted from the data. Unlike the Fourier-based tools which map signals onto a fixed basis set, EMD is a unique signal decomposition tool that makes no prior assumptions about the data.

A significant feature of the EMD analysis is its dependency on only the data adaptively and its capacity of finding appropriate basis functions that may bring out the important information in signal. Actually, different IMFs may have different physical meaning especially valid for practical applications so one of the potential future plan is to determine the existence of this meaning.

In the experimental evaluations we test classification performance of the mRMR feature selection scheme both before and after the EMD based signal decomposition. We observed significant performance improvements in the decomposed representation with mRMR feature selection. While the classification accuracy of the baseline system was $71.6 \%$, it improved to $79.4 \%$ using feature selection in the decomposed representation. This is an encouraging improvement, which indicates that the EMD based multi-resolution analysis creates a richer set of features and the mRMR successfully selects a smaller set of relevant features among them.

The technique utilized here is a high-resolution method used as an alternative to Fourier-based analysis, which seem as the standard technique especially for speech signals. However, a comparative study considering other time-frequency tools such as spectrogram or autoregressive models is needed in the study of dietary monitoring to better interpret the pros and cons of them. Aside from such a study, this research has shown that the EMD has a promising application in the analysis of intake classification despite of the fact that it doesn't provide a complete theory rather an empirical analysis.

One limitation of our evaluation is the small dataset size. In future work, we plan to collect free-living data with longer duration (e.g. entire day or more) recordings over an extended set of food types and subjects. It would be also of interest to find new feature descriptors which can improve the discrimination performance. 


\section{REFERENCES}

[1] SM Shafiul Alam and Mohammed Imamul Hassan Bhuiyan. 2013. Detection of seizure and epilepsy using higher order statistics in the EMD domain. IEEE Journal of Biomedical and Health Informatics 17, 2 (2013), 312-318.

[2] Oliver Amft, Holger Junker, and Gerhard Troster. 2005. Detection of eating and drinking arm gestures using inertial body-worn sensors. In Wearable Computers, 2005. Proceedings. Ninth IEEE International Symposium on. IEEE, 160-163.

[3] Michael A Crary, Jamie L Humphrey, Giselle Carnaby-Mann, Raam Sambandam, Leslie Miller, and Scott Silliman. 2013. Dysphagia, nutrition, and hydration in ischemic stroke patients at admission and discharge from acute care. Dysphagia 28, 1 (2013), 69-76.

[4] Ingrid Daubechies. 1988. Orthonormal bases of compactly supported wavelets. Communications on pure and applied mathematics 41, 7 (1988), 909-996.

[5] Chris Ding and Hanchuan Peng. 2005. Minimum redundancy feature selection from microarray gene expression data. Fournal of bioinformatics and computational biology 3, 02 (2005), 185-205.

[6] Cumhur Ertekin, Nur Yüceyar, et al. 1998. Clinical and electrophysiological evaluation of dysphagia in myasthenia gravis. fournal of Neurology, Neurosurgery \& Psychiatry 65, 6 (1998), 848-856.

[7] Patrick Flandrin, Gabriel Rilling, and Paulo Goncalves. 2004. Empirical mode decomposition as a filter bank. IEEE Signal Processing Letters 11, 2 (2004), 112-114.

[8] Leela K Gudupudi, Navin Chatlani, Christophe Beaugeant, and Nicholas Evans. 2015. Non-linear acoustic echo cancellation using empirical mode decomposition. In Acoustics, Speech and Signal Processing (ICASSP), IEEE International Conference on. IEEE, 614-618.

[9] Daniel Harari, Miriam Furst, Nahum Kiryati, Asaf Caspi, and Michael Davidson. 2001. A computer-based method for the assessment of body-image distortions in anorexia-nervosa patients. IEEE Transactions on Information Technology in Biomedicine 5, 4 (2001), 311-319.

[10] Norden E Huang, Zheng Shen, et al. 1998. The empirical mode decomposition and the Hilbert spectrum for nonlinear and non-stationary time series analysis. In Proceedings of the Royal Society of London A: Mathematical, Physical and
Engineering Sciences, Vol. 454. The Royal Society, 903-995.

[11] Tal Klap and Zvika Shinar. 2013. Using piezoelectric sensor for continuouscontact-free monitoring of heart and respiration rates in real-life hospital settings. In Computing in Cardiology Conference (CinC), 2013. IEEE, 671-674.

[12] M-È Labonté, A Cyr, L Baril-Gravel, MM Royer, and B Lamarche. 2012. Validity and reproducibility of a web-based, self-administered food frequency questionnaire. European journal of clinical nutrition 66, 2 (2012), 166-173.

[13] David Lansky and Kelly D Brownell. 1982. Estimates of food quantity and calories: errors in self-report among obese patients. The American journal of clinical nutrition 35, 4 (1982), 727-732.

[14] Danilo P Mandic, Naveed ur Rehman, Zhaohua Wu, and Norden E Huang. 2013. Empirical mode decomposition-based time-frequency analysis of multivariate signals: The power of adaptive data analysis. IEEE signal processing magazine 30 , 6 (2013), 74-86.

[15] Xuan Vinh Nguyen, Jeffrey Chan, Simone Romano, and James Bailey. 2014. Effective global approaches for mutual information based feature selection. In Proceedings of the international conference on knowledge discovery and data mining. ACM, 512-521.

[16] World Health Organization. 2017. Obesity and overweight. (2017). http://www. who.int/mediacentre/factsheets/fs311/en [Accessed 6-March-2017].

[17] Edward S Sazonov and Juan M Fontana. 2012. A sensor system for automatic detection of food intake through non-invasive monitoring of chewing. IEEE sensors journal 12, 5 (2012), 1340-1348.

[18] M. A. Tuğtekin Turan and Engin Erzin. 2013. Enhancement of throat microphone recordings by learning phone-dependent mappings of speech spectra. In Acoustics, Speech and Signal Processing (ICASSP), IEEE International Conference on. IEEE, 7049-7053.

[19] M. A. Tuğtekin Turan and Engin Erzin. 2016. Source and filter estimation for throat-microphone speech enhancement. IEEE/ACM Transactions on Audio, Speech, and Language Processing 24, 2 (2016), 265-275.

[20] Luc F Van Gaal, Ilse L Mertens, and E Christophe. 2006. Mechanisms linking obesity with cardiovascular disease. Nature 444, 7121 (2006), 875-880. 